\title{
Assistência aos pacientes que desenvolveram miocardite pós-infecção do COVID-19
}

\author{
Assistance to patients who developed COVID-19 post-infection myocarditis
}

Asistencia a los pacientes que desarrollaron miocarditis posterior a la infección de COVID19

Maria Luíza Alves Guerra ${ }^{1 *}$, Camila Carvalho Rodrigues Costa ${ }^{2}$, Daniela Ferrari Angelo Ferreira ${ }^{3}$, Davi Heringer Coelho Bisi ${ }^{4}$, Elisa Almeida Rezende ${ }^{5}$, Ludmilla Isadora Mendes Fagundes ${ }^{6}$, Mariana Vanon Moreira ${ }^{5}$, Pauline Christina Campos Martins Ferreira ${ }^{7}$, Tiago Picolo Fernandes ${ }^{8}$, Humberto de Freitas Boy?.

\section{RESUMO}

Objetivo: Investigar, interpretar e revisar os achados sobre o desenvolvimento do fenótipo de miocardite viral após infecção por SARS-CoV-2, bem como os mecanismos fisiopatológicos envolvidos, para que seja possível a formulação de estratégias terapêuticas para prevenir e atenuar a evolução do quadro clínico. Revisão bibliográfica: Observa-se que os pacientes com piores prognósticos são os idosos ou aqueles que apresentam hipertensão arterial sistêmica, diabetes mellitus, doença arterial coronariana e insuficiência cardíaca. Estudos mostram que cerca de 10\% dos pacientes infectados por COVID-19 desenvolveram miocardite e que $59 \%$ dos doentes com acometimento cardiovascular evoluíram para óbito intra-hospitalar. Isso se deve porque a COVID-19 causa lesão miocárdica que se manifesta por elevação dos níveis de troponina relacionando-se com o aumento da mortalidade. Outros marcadores de inflamação podem ser encontrados, como DHL, dímero-D, IL-6 e ferritina. A interação entre SARS-CoV e os receptores ACE-2 pode colaborar com a inflamação aumentando o dano no miocárdio. Considerações finais: $O$ manejo dos pacientes com miocardite, é realizado por meio da utilização de imunossupressor e antiviral a fim de suprimir as respostas inflamatórias e autoimunes.

Palavras-chave: Covid-19, Miocardite, Coronavírus.

\begin{abstract}
Objective: To investigate, interpret and review the findings on the development of the viral myocarditis phenotype after SARS-CoV-2 infection, as well as the pathophysiological mechanisms involved, so that it is possible to formulate therapeutic strategies to prevent and mitigate the evolution of the condition clinical. Bibliographic review: It is observed that the patients with the worst prognosis are the elderly or those who have systemic arterial hypertension, diabetes mellitus, coronary artery disease and heart failure. Studies show that around $10 \%$ of patients infected with COVID-19 developed myocarditis and that $59 \%$ of patients with cardiovascular involvement progressed to in-hospital death. This is because COVID-19 causes a myocardial injury that manifests itself by an increase in troponin levels, which is related to an increase in mortality. Other inflammation markers can be found, such as DHL, D-dimer, IL-6 and ferritin. The interaction between SARS$\mathrm{CoV}$ and ACE-2 receptors can contribute to inflammation by increasing myocardial damage. Final considerations: The management of patients with myocarditis is performed through the use of immunosuppressants and antiviral drugs in order to suppress inflammatory and autoimmune responses.
\end{abstract}

Key words: Covid-19, Myocarditis, Coronavirus.

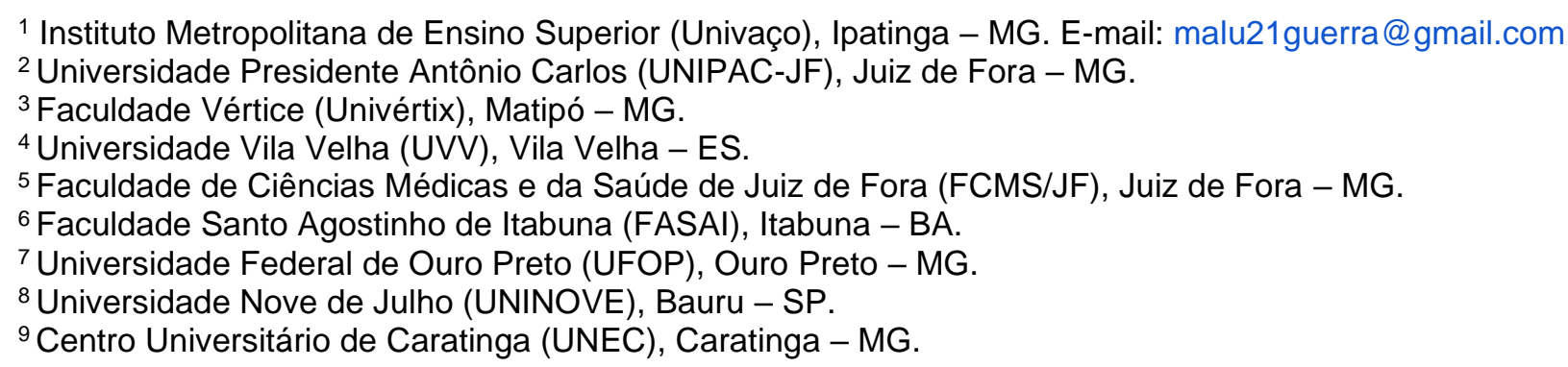




\section{RESUMEN}

Objetivo: Investigar, interpretar y revisar los hallazgos sobre el desarrollo del fenotipo de miocarditis viral luego de la infección por SARS-CoV-2, así como los mecanismos fisiopatológicos involucrados, de manera que sea posible formular estrategias terapéuticas para prevenir y mitigar la evolución de la condición clínica. Revisión bibliográfica: Se observa que los pacientes con peor pronóstico son los ancianos o los que presentan hipertensión arterial sistémica, diabetes mellitus, enfermedad arterial coronaria e insuficiencia cardíaca. Los estudios muestran que alrededor del 10\% de los pacientes infectados con COVID-19 desarrollaron miocarditis y que el $59 \%$ de los pacientes con afectación cardiovascular evolucionaron hasta la muerte intrahospitalaria. Esto se debe a que COVID-19 provoca una lesión miocárdica que se manifiesta por un aumento de los niveles de troponina relacionado con el aumento de la mortalidad. Se pueden encontrar otros marcadores de inflamación, como DHL, dímero D, IL-6 y ferritina. La interacción entre los receptores SARS-CoV y ACE-2 puede contribuir a la inflamación al aumentar el daño miocárdico. Consideraciones finales: El manejo de los pacientes con miocarditis se realiza mediante el uso de inmunosupresores y fármacos antivirales con el fin de suprimir las respuestas inflamatorias y autoinmunes.

Palabras clave: Covid-19, Miocarditis, Coronavirus.

\section{INTRODUÇÃO}

A Covid-19 é uma Síndrome Respiratória Aguda Grave (SARS-CoV-2) que surgiu em dezembro de 2019 na cidade de Wuhan, província de Hubei, localizada na China. O novo coronavírus descoberto se trata de um vírus pertencente à família, já conhecido pelos cientistas, dos coronavírus que cursam com patologias do trato respiratório como, pneumonia e resfriado comum. Foi rapidamente declarada uma pandemia e preocupação global devido à sua rápida transmissibilidade através de gotículas de saliva e secreções presentes no ar após um indivíduo contaminado tossir ou espirrar próximo dos não contaminados. Por se tratar de uma síndrome gripal, seus principais sintomas incluem febre, mialgia, tosse seca ou produtiva, cefaleia e dispneia, sendo este último sintoma o de maior destaque juntamente com a febre acima de 37,5 graus Celsius registrada (ROMANO E, 2021; OZIERANSKI K, et al., 2021; ASKIN L, et al., 2020; FERRARI F, 2020; FIGUEIREDO NETO JA, et al., 2020).

Embora os pulmões sejam os primeiros órgãos a serem atingidos, o coração também possui papel importante nesses casos, uma vez que é acometido em muitos casos e contribui para um pior prognóstico da doença, sendo visto em $22 \%$ dos pacientes hospitalizados. À vista dos fenômenos provenientes da infecção viral pelo novo coronavírus, a denominação "síndrome cardiovascular aguda pela COVID-19" vem sendo proposta para descrever as alterações sindrômicas no sistema cardiovascular após infecção confirmada pela COVID-19. O principal grupo populacional que apresenta maiores acometimentos cardíacos é o dos idosos, juntamente com os pacientes que já tenham hipertensão arterial sistêmica, diabetes mellitus, doença arterial coronariana e insuficiência cardíaca. Sendo assim, são estes pacientes que necessitam de um maior aporte ventilatório e caso haja piora do quadro, frequentemente progridem ao óbito (FEITOSA G, et al., 2020; PERILLO FILHO M, et al., 2020; FIGUEIREDO NETO JA, et al., 2020; ROMANO E, 2021; GOLDRAICH L, et al., 2020).

O acometimento cardíaco nos doentes com o novo coronavírus resultam da injúria miocárdica que envolvem processos miocárdicos não isquêmicos, incluindo infecção respiratória grave com hipóxia, assim como pode resultar a partir da sepse, inflamação sistêmica e tromboembolismo pulmonar. Dentre as doenças citadas, a miocardite tem sido encontrada em torno de $7 \%$ dos pacientes que testaram positivo para COVID19. Como a SARS-COV-2 promove inflamação sistêmica, tanto a ação pró-coagulante, quanto a ação de disrupção autoimune podem ser desencadeadas levando à lesão miocárdica grave no paciente (COSTA IBSS, et al., 2020; FIGUEIREDO NETO JA., et al., 2020; MAMADE Y, et al., 2020; PERILLO FM., et al., 2020; ROMANO E, 2021).

Dessa forma, fenômenos inflamatórios miocárdicos derivados da infecção viral por COVID-19 são comuns e com piores desfechos, podem progredir para remodelação cardíaca, disfunção miocárdica e óbito. Assim, diante do contexto, surge a questão problema de como seria o diagnóstico e manejo da miocardite em pacientes infectados previamente com COVID 19 (COSTA IBSS, et al., 2020; PERILLO FM, et al., 2020). 
Sendo assim, o presente artigo teve como objetivo ratificar, por meio de uma revisão narrativa, a assistência aos pacientes que desenvolveram miocardite viral após infecção por SARS-CoV-2, bem como os mecanismos fisiopatológicos envolvidos, para que seja possível a formulação de estratégias terapêuticas que possam prevenir e atenuar a evolução do quadro clínico mencionado devido a uma maior suscetibilidade de pior prognóstico.

\section{REVISÃO BIBLIOGRÁFICA}

\section{Etiologia do novo coronavírus e epidemiologia de pacientes com doenças cardiovasculares por complicações da covid-19}

No final de 2019, um novo membro da família Coronaviridae, SARS-CoV-2, foi identificado em um grupo de pacientes com pneumonia em Wuhan. Ele se espalhou rapidamente, resultando em uma epidemia em toda a China, seguida por uma pandemia global. O SARS-CoV-2 recebeu este nome por pertencer ao gênero Betacoronavírus da família Coronaviridae, é o sétimo coronavírus a infectar seres humanos (ROMANO E, 2021; GORBALENYA AE, et al., 2020; PERILLO FM, et al., 2020).

Seu primeiro nome, SARS, é uma abreviação para Síndrome Aguda Respiratória Severa, que traz consigo um dos principais sintomas clínicos da forma grave de inúmeras doenças respiratórias, a dispneia. Além disso, CoV é uma abreviação para a família à qual pertence, Coronaviridae, e o número 2 representa a segunda espécie de coronavírus por ter um material genético semelhante ao SARS-CoV que também quase iniciou uma pandemia no século XXI. Um dos estudos iniciais foi realizado pelo Centro Chinês para Controle e Prevenção de Doenças que avaliou o grau de gravidade de 72.314 pacientes com Covid-19 nessa população. Em $81,4 \%$ dos casos a doença foi classificada como leve, grave em 13,9\% e crítica em 4,7\% (ROMANO E, 2021; GORBALENYA AE, et al., 2020; PERILLO FM, et al., 2020; RANARD LS, et al., 2020).

Por mais que o novo coronavírus apresenta-se clinicamente como uma síndrome gripal, àqueles pacientes que apresentam comorbidades prévias ou alto risco cardiovascular, como idade avançada, sistema imunológico comprometido, terem predisposição a DCV ou por apresentarem níveis elevados de ECA2 no organismo, tendem a apresentar injúria miocárdica e outros acometimentos cardíacos. Os pacientes evoluíam com piores prognósticos e viram que eram mais idosos ou que apresentavam hipertensão arterial sistêmica, diabetes mellitus, doença arterial coronariana e insuficiência cardíaca como antecedente pessoal (COSTA IBSS, et al., 2020; ASKIN I, et al., 2020; ROMANO E, 2021; VALENZUELA-RODRIGUEZ G e AMADO-TINEO $P, 2019)$.

Entre 22\% a 31\% dos pacientes eram admitidos nas Unidades de Terapia Intensiva (UTI) e necessitavam de um maior suporte ventilatório, assim como, até 59\% dos doentes com acometimento cardiovascular evoluíram para óbito intra-hospitalar. Estudos também mostraram que cerca de 10\% dos casos relatados de COVID-19 ao redor do mundo desenvolveram miocardite, principalmente os pacientes mais críticos e com muitas comorbidades (COSTA IBSS, et al., 2020; ASKIN I, et al., 2020; ROMANO E, 2021).

\section{Mecanismos de lesão miocárdica: fisiopatologia da lesão e envolvimento com o covid-19}

A lesão miocárdica se manifesta por elevação dos níveis de troponina, acima do percentil 99 do limite normal, e devido a isso, está associada ao aumento da mortalidade, podendo ocorrer juntamente com alterações eletrocardiográficas e ecocardiográficas. Estudiosos observaram que tais manifestações miocárdicas descritas foram a partir de pacientes com altas cargas virais e, que em alguns casos uma progressão do quadro para uma miocardite fulminante (OZIERANSKI K, et al., 2021; ROMANO E, 2021).

De acordo com a Sociedade Europeia de Cardiologia (ESC), a miocardite pode ser causada por muitos agentes infecciosos, não infecciosos e também pode ser o resultado de um processo autoimune. Por ser uma patologia de acometimento cardíaco, pode se manifestar com uma vasta sintomatologia clínica, como, dor torácica agudizada, dispneia aos pequenos e médios esforços, assim como, pode apresentar sinais eletrocardiográficos de arritmias ou disfunção ventricular esquerda aguda nos pacientes com miocardite. 0 material genético viral pode ser encontrado em até dois terços dos casos de miocardite ou com cardiomiopatia dilatada (BABAPOOR-FARROKHRAN S, et al., 2020; ADÃO R e GUZIK TJ, 2020; ROMANO E, 2021). 
Estudiosos relatam que a infecção humana por SARS-COV no miocárdio é conhecida por depender dos receptores ACE-2, em que a interrupção da ACE-2 leva a uma cardiomiopatia dependente da idade, disfunção cardíaca e insuficiência cardíaca. Sugeriram que a interação entre SARS-CoV e ACE-2 no coração poderia colaborar à inflamação, dano miocárdico mediado por SARS, do mesmo modo que, observaram relevante infiltração de macrófagos no miocárdio em amostras de coração post-mortem. Além desse fato, foi detectado RNA viral SARS-CoV em amostras de coração humano autopsiadas, sugerindo invasão direta de fragmentos do vírus, juntamente com a redução acentuada da regulação da ACE-2 e reduções na proteína ACE-2 nas amostras cardíacas (ADÃO R e GUZIK TJ, 2020; BABAPOOR-FARROKHRAN S, et al., 2020; ROMANO E, 2021; KOCIOL RD, et al., 2020).

O efeito prejudicial da regulação negativa de ACE-2 impediria os efeitos cardioprotetores de angiotensina 1-7 levando ao aumento da produção de TNFa. O TNFa é uma citocina inflamatória comum e muitos pesquisadores demonstraram que a resposta inflamatória pode ser pelo menos parcialmente responsável pelo dano miocárdico. A resposta inflamatória acentuada pode gerar coagulação intravascular disseminada (DIC) (ADÃO R e GUZIK TJ, 2020; BABAPOOR-FARROKHRAN S, et al., 2020; ROMANO E, 2021; KOCIOL $\mathrm{RD}$, et al., 2020).

Devido à semelhança significativa da infecção por SARS-CoV com SARS-CoV-2, os possíveis mecanismos de lesão miocárdica podem ser danos diretos ao coração, mas também pode causar fibrose intersticial miocárdica, inflamação sistêmica, desestabilização da placa coronariana e até hipóxia. Nesse contexto, quadros clínicos de tromboembolismo pulmonar têm sido descritos, e postula-se que a trombose microvascular seria outro mecanismo com alto potencial para lesão miocárdica (FEITOSA G, et. al., 2020; XAVIER AR, et al., 2020; OZIERANSKI K, et al., 2021; ROMANO E, 2021).

Os mecanismos fisiopatológicos subjacentes à lesão miocárdica causada por COVID-19 não são bem conhecidos até o momento, requerendo mais estudos aprofundados para delinear melhor esse processo lesivo. Assim, mesmo que haja inúmeras infecções por SARS-CoV-2 que causem alguma injúria cardíaca, ainda não há provas concretas de que o SARS-CoV-2 seja um novo vírus cardiotrópico e que cause danos direto no músculo cardíaco. Devido a isso, há vários estudos que destacam a importância de medidas agressivas de rastreamento em populações de alto risco para estabelecer o diagnóstico em tempo hábil e poder evitar consequências ou agressões ainda piores ao coração dos pacientes infectados e apresentar um melhor prognóstico frente ao COVID-19 (XAVIER AR, et al., 2020; ROMANO E, 2021; LONG B, et al., 2020; RANARD LS, et al., 2020; KOCIOL RD, et al., 2020).

\section{Apresentação clínica e diagnóstico}

A COVID-19, por ser um vírus de RNA envelopado, apresenta uma clínica semelhante às outras infecções virais na qual estabelece sintomas comuns como tosse seca ou produtiva, febre, dispneia, mialgia e congestionamento nasal, assim como, pode apresentar sintomas menos comuns, por exemplo queixas gastrointestinais, anosmia, ageusia, cefaleia e conjuntivite. A clínica do paciente acometido pelo vírus é dominante por sintomas respiratórios, todavia, podem ocasionar complicações cardiovasculares. Dentre os pacientes com COVID-19, há muitos que evoluem com bom prognóstico, em contrapartida, aqueles que apresentam quadros de maior risco, tendem a evoluir conforme a idade avançada e comorbidades relacionadas (FEITOSA G, et al., 2020; XAVIER AR, et al., 2020; VALENZUELA-RODRIGUEZ G e AMADOTINEO P, 2019; KOCIOL RD, et al., 2020).

Ao analisar as complicações cardiovasculares nos pacientes com. COVID-19, temos como principais manifestações a miocardite, insuficiência cardíaca descompensada, síndrome coronária aguda (SCA), choque cardiogênico e infecção em um coração transplantado. Levando em consideração que ainda é algo reverente e com muitas incertezas a respeito das consequências do SARS-CoV-2 no organismo, sabe-se que miocardite pode estar presente em pacientes que já apresentam um elevado risco cardiovascular, assim como pode condicionar a uma lesão miocárdica aguda e aumento da gravidade do quadro, o que leva a uma maior necessidade em internação nesses casos graves (DENG Q, et al., 2020; MAMADE Y, et al., 2020; RANARD LS, et al., 2020). 
O diagnóstico da miocardite é feito por meio de Biópsia Endomiocárdica (BEM), mas existem vários testes laboratoriais para confirmar que são feitos de forma seriada nos pacientes internados a fim de verificarem a evolução do quadro, são eles a dosagem sérica de Troponina I e peptídeo natriurético cerebral (NT-pro BNP), Ecocardiograma e Eletrocardiograma (ECG). O ECG costuma ter alterações, como baixa voltagem de QRS, distúrbios de ritmo, segmento ST, anormalidades na onda T e alterações na condução (AGDAMAG AC, et al., 2020; RANARD LS, et al., 2020).

Além desses exames, há a Ecocardiografia Transtorácica (ETT) que avalia as complicações cardíacas associadas ao vírus, podendo encontrar edema miocárdico, disfunção ventricular esquerda ou biventricular global, pericárdio e trombo de VE. Assim como, há a Ressonância Magnética Cardíaca (RMC) que avalia a estrutura da função biventricular, no qual pode-se estabelecer diagnóstico de edema do miocárdio e fibrose intersticial. Entretanto, ainda que tais exames sejam realizados para diagnóstico de miocardite, muitas vezes não apresentam alta especificidade para confirmar tal patologia e, em contrapartida, apresentam alta sensibilidade que são observadas em outras complicações cardiovasculares além da miocardite (AGDAMAG AC, et al., 2020; DENG Q, et al., 2020; LONG B, et al., 2020; VALENZUELA-RODRIGUEZ G e AMADOTINEO P, 2019).

\section{Manejo}

Em casos de infecção por SARS-COV-2, a conduta clínica a ser adotada varia conforme a gravidade do quadro. A maioria dos pacientes com nenhum ou poucos sintomas e que são estáveis clinicamente dispensam internação hospitalar. Já os pacientes que apresentam sintomas mais graves e com prognóstico desfavorável requerem internação (COSTA IBSS, et al., 2020; DENG Q, et al., 2020; MAMADE Y, et al., 2020).

$\mathrm{O}$ tratamento dos pacientes que testaram positivo ao novo coronavírus varia também em relação ao risco cardiovascular que cada um apresenta, assim como, é levado em consideração as comorbidades geradas pelo vírus. A miocardite, como uma das principais consequências do COVID-19, requer terapêutica imunossupressora e antiviral de modo a suprimir respostas inflamatórias e autoimunes. A ação dos fármacos a miocardite propicia a melhora da clínica, da função ventricular e, consequentemente, a não replicação e eliminação do vírus (ARAGÃO CAS, et al., 2020; ROMANO E, 2021; VALENZUELA-RODRIGUEZ G e AMADO-TINEO P, 2019).

Entre as vias de tratamento há a infusão subcutânea de Interferon-beta (IFN-B) e a Imunoglobulina Intravenosa (IG-IV). Outro caminho terapêutico é a administração de pentoxifilina, que bloqueia a transcriptase do TNF-alfa e diminui níveis de interleucinas 2 e 6 , reduzindo efeitos cardio-agressivos das citocinas e também pode atuar como anti-inflamatório (ARAGÃO CAS, et al., 2020; SIRIPHANTHONG B, et al., 2020).

A American Heart Association (AHA) recomenda a implementação do protocolo para choque cardiogênico, o que inclui a administração de inotrópicos e outras medidas dependendo do quadro do paciente como, vasopressores ventilação mecânica e até mesmo oxigenação por membrana extracorpórea por meio de um dispositivo de assistência ventricular ou bomba de Balão Intra-Aórtico (BIA). Já a European Society of Cardiology (ESC) não endossou o uso de imunoglobulina intravenosa devido à falta de evidências de apoio, e desencorajou o uso de corticoides na miocardite com infecção ativa, citando a ineficácia de corticosteroides de um ensaio clínico randomizado. Somado a isso, sabe-se que a imunossupressão pode representar riscos de agravamento da doença, especialmente durante a replicação viral ativa do vírus (SIRIPHANTHONG B, et al., 2020; DENG Q, et al., 2020; MAMADE Y, et al., 2020; ÇINAR T, et al., 2020).

Por mais que o tratamento dependa da clínica de cada indivíduo, o monitoramento cardíaco é recomendado para possibilitar terapia apropriada para bradicardia e taquiarritmias, incluindo bloqueio atrioventricular e taquicardia ventricular ou fibrilação atrial. As bradiarritmias podem exigir estimulação cardíaca temporária e as taquiarritmias podem responder a medicamentos antiarrítmicos, como lidocaína e mexiletina (WU CL, et al., 2020; ROMANO E, 2021; RANARD LS, et al., 2020).

\section{CONSIDERAÇÕES FINAIS}

Por meio da análise bibliográfica, verificou-se que a miocardite decorrente da infecção por SARS-CoV-2 deve-se ao aumento dos fatores pró inflamatórios de forma sistêmica. Assim, lesa diretamente o sistema 
cardiovascular e o miocárdio, aumentando os valores de troponina e interação entre SARS-CoV e receptores ACE-2. Além disso, a progressão da injúria miocárdica está relacionada proporcionalmente com a idade e os riscos cardiovasculares de cada paciente, de forma que o tratamento se torne variado e de acordo com a gravidade do quadro. Portanto, a fim de proporcionar o manejo apropriado da miocardite, torna-se necessário maiores estudos e individualização de cada paciente devido às variedades terapêuticas que possam ser consideradas como monitorização cardíaca, uso de imunossupressores e outros fármacos que diminuem a resposta inflamatória e evitem a progressão clínica.

\section{REFERÊNCIAS}

1. ADÃO R, GUZIK TJ. Inside the heart of COVID-19. Cardiovasc Research and Development Center, 2020; 116(6): 5961.

2. AGDAMAG ACC, et al. Update on COVID-19 Myocarditis. Multidisciplinary Digital Publishing Institute, 2020; 678(56): 1-10.

3. ARAGÃO CAS, et al. Miocardite pelo SARS-CoV-1: Existem diferenças com relação a outras Miocardite Virais?. Revista Sociedade de Cardiologia do Estado de São Paulo, 2020, 30(4): 23-27.

4. ASKIN L, et al. O Efeito da Doença de Coronavírus 2019 nas Doenças Cardiovasculares. Arq Bras Cardiol, 2020; 114(5): 817-822.

5. BABAPOOR-FARROKHRAN S, et al. Myocardial injury and COVID-19: Possible mechanisms. Life Sciences, 2020; 253: 117723.

6. COSTA IBSS, et al. O Coração e a COVID-19: O que o Cardiologista Precisa Saber. Arq. Bras. Cardiol. 2020; 114(5): 805-816.

7. DENG Q, et al. Suspected myocardial injury in patients with COVID-19: Evidence from front-line clinical observation in Wuhan, China. International Journal of Cardiology. 2020; 311: 116-121.

8. FEITOSA G, et al. COVID-19 e o Coração. Rev. Cient. HSI 2020; 4(2): 77-88.

9. FIGUEIREDO NETO JA, et al. Doença de Coronavírus-19 e o Miocárdio. Arquivos Brasileiros de Cardiologia. 2020; 114 (6): 1051-1057.

10. FERRARI F. COVID-19: Dados Atualizados e sua Relação Com o Sistema Cardiovascular. Arq Bras Cardiol, 2020; 114(5): 823-826.

11. GOLDRAICH LA, et al. Tópicos Emergentes em Insuficiência Cardíaca: COVID-19 e Insuficiência Cardíaca. Arq Bras Cardiol. 2020; 115(5): 942-944.

12. GORBALENYA AE, et al. Coronaviridae Study Group of the International Committee on Taxonomy of Viruses., The species Severe acute respiratory syndrome-related coronavirus: classifying 2019-nCoV and naming it SARS-CoV-2. Nat Microbiol 2020; (5): 536-544.

13. KOCIOL RD, et al. Recognition and initial management of fulminant myocarditis: a scientific statement from the American Heart Association. Circulation 2020; 141: 69-92.

14. LONG B, et al. Cardiovascular complications in COVID-19. American Journal Of Emergency Medicine, 2020.

15. MAMADE Y, et al. COVID-19 e Doença Cardiovascular: Consequências Diretas e Linhas de Investigação. Revista da Sociedade Portuguesa de Medicina Interna. 2020; 27(3): 257-263.

16. OZIERANSKI K, et al. Clinically suspected myocarditis in the course of severe acute respiratory syndrome novel coronavirus-2 infection: fact or fiction?. Journal of Cardiac Failure. 2021; 27(1): 92-96.

17. PERILLO FM, et al. Esporte em Tempos de Covid-19: Alerta ao Coração. Arq Bras Cardiol. 2020; $115(3): 303-307$.

18. RANARD LS, et al. Approach to Acute Cardiovascular Complications in COVID-19 Infection. Circ Heart Fail, 2020; 13: 007220.

19. ROMANO E. Associação de Injúria Miocárdica e Mortalidade em Pacientes Hospitalizados com COVID-19. Arq Bras Cardiol, 2021; 116(2): 283-284.

20. SIRIPHANTHONG B, et al. Recognizing COVID-19-related myocarditis: The possible pathophysiology and proposed guideline for diagnosis and management. Heart Rhythm. 2020. 17(9): 1463-1471.

21. VALENZUELA-RODRIGUEZ G, AMADO-TINEO P. Compromiso cardiovascular en COVID-19. Rev Soc Peru Med Interna. 2019; 33(2): 61-67.

22. VERMA V, et al. COVID-19 associated viral myocarditis: does it exist? Monaldi Archives for Chest Disease, 2020; (90): 1382.

23. XAVIER AR, et al. COVID-19: manifestações clínicas e laboratoriais na infecção pelo novo coronavírus. J Bras Patol Med Lab, 2020; (56): 1-9.

24. WU CL, et al. SARS-CoV-2, COVID-19, and inherited arrhythmia syndromes. Heart Rhythm 2020; $17: 1456-1462$.

25. ÇINAR T, et al. COVID-19 and acute myocarditis: current literature review and diagnostic challenges. Revista da Associação Médica Brasileira 2020; 66(2): 48-54. 